\title{
El valor de lo Sagrado: Percepción y espiritualidad en torno al concepto común de Patrimonio Arquitectónico
}

\author{
Jonathan Ruiz-Jaramillo* María Dolores Joyanes-Díaz** \\ Universidad de Málaga (España)
}

\begin{abstract}
Resumen: Desde la constitución de las primeras comunidades, la materialización en el territorio de acontecimientos sociales, rituales o ceremonias ha significado una progresiva asociación del lugar y del paisaje a valores trascendentales específicos. Con el racionalismo moderno, la escisión entre sujeto y objeto motivó el abandono progresivo de estos valores inherentes a estos enclaves. A partir de un conjunto de ejemplos se propone una reflexión sobre los valores intangibles e inmateriales ligados a espacios que a lo largo de la historia han sido el centro de prácticas discursivas y ritos simbólicos. Trascendiendo la configuración material arquitectónica han quedado incorporados como componentes inseparables del concepto de patrimonio. Estas cualidades intangibles deben ser consideradas en los procesos de rehabilitación y puesta en valor de manera que prevalezca la identidad social mediante su adaptación a cada época, independientemente de la alternancia sucesiva de culturas a lo largo de su historia.
\end{abstract}

Palabras Clave: Sociedad; Religión; Espacio; Arquitectura; Patrimonio.

Value of the Sacred: Perception and spirituality around the common concept of Architectural Heritage

\begin{abstract}
Since the formation of the first communities, the materialization in the territory of social events, rituals or ceremonies has signified a progressive association of the site and the landscape with specific transcendental values. With modern rationalism, the division between subject and object motivated the progressive abandonment of these values inherent to these enclaves. From a set of examples, a reflection is proposed on the intangible and immaterial values linked to spaces that throughout history have been the centre of discursive practices and symbolic rites. Transcending the material architectural configuration, these have been incorporated as inseparable components of the global concept of heritage. These intangible qualities must be considered in the restoration and enhancement processes to ensure the prevalence of social identity through their adaptation to each period, regardless of the successive alternation of cultures throughout their history.
\end{abstract}

Keywords: Society; Religion; Space; Architecture; Heritage.

\section{La identidad personal y su vinculación con el concepto de sagrado}

Desde la generación consciente de los primeros grupos sociales primitivos, el hombre reconoció una serie de valores que trascendían la mera objetividad cotidiana, los cuales estarían asociados a una concepción primaria y elemental de lo sagrado, independientemente de la dimensión o profundidad que este concepto pudiese alcanzar (Falk and Gillespie 2009). Así, cuando el ser humano habitaba en las cuevas prehistóricas, determinados espacios de su interior e incluso cuevas completas se dotaron de un significado que trascendía el meramente funcional. Estos quedaban ligados inequívocamente a la emocionalidad, trascendencia y espiritualidad de estas incipientes sociedades. Los ritos asociados a ellas conectaban a las gentes con estos espacios, los cuales, como lugares de encuentro y comunión, eran capaces de fortalecer creencias compartidas a través de la socialización (Mazumdar and Mazumdar 1993).

\footnotetext{
* Universidad de Málaga (España); E-mail: jonaruizjara@uma.es; https://orcid.org/0000-0002-2527-9170

**Universidad de Málaga (España); E-mail: lolajoyanes@uma.es; http://orcid.org/0000-0002-0773-2381
} 
El primer refugio como aislamiento respecto a las fuerzas de la naturaleza ya implicaba, por su condición separada del resto, la asignación a este del concepto de sagrado por parte del grupo. En él podían sentirse a salvo, en la intimidad que le proporcionaba el umbral de entrada que establecía la diferencia entre exterior e interior, entre luz y oscuridad, entre lo desconocido y lo controlado. En efecto, el propio diccionario de la Lengua Española identifica aún el concepto de sagrado con "Huir de una dificultad acogiéndose a una determinada protección" (Real Academia Española 2017). Al mismo tiempo, los hitos de la naturaleza y sus diferentes manifestaciones, con su papel asociado a objetos de adoración, extendían el concepto y transformaban en sagrado el entorno circundante. Trascendiendo los primeros refugios naturales, conectada con la propia evolución social, comenzaron a erigirse estructuras específicas ligadas a ritos ceremoniales. Así surgen estructuras megalíticas como Göbekli Tepe en Turquía ( $\approx 9000$ a.C.) o Ggantija en Gozo (Malta) ( $\approx 3600$ a.C.) (Figura 1: Conjunto megalítico Ggantija en la isla de Gozo (Malta)), consideradas como los centros espirituales o templos más antiguos de la humanidad encontrados hasta el momento (Atakuman 2014).

\section{Figura 1: Conjunto megalítico Ggantija en la isla de Gozo (Malta)}
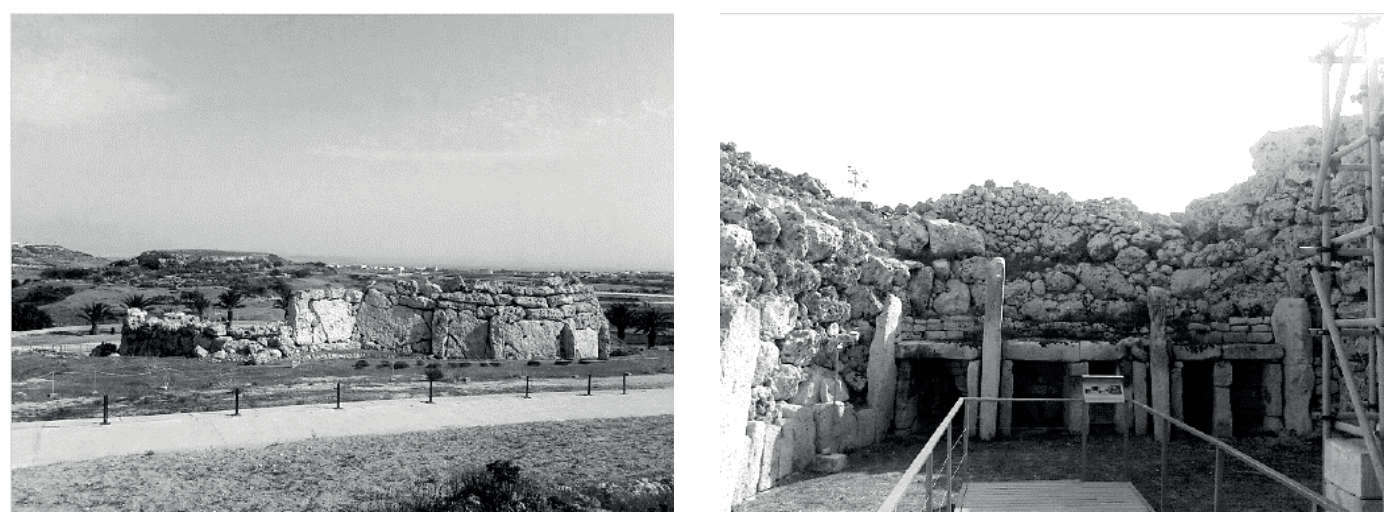

Estas formas arquitectónicas primitivas constituidas en lugares para el culto relacionados habitualmente con el fin de la vida, en forma de enterramientos, se erigen además a la manera de signos visibles en el territorio, como señal indicadora de itinerarios o delimitadora de dominios. Su propia concepción espacial está ligada a su percepción del mundo, de la realidad física y sus fenómenos naturales asociados, a los que buscaban dar sentido. Ello tiene una implicación directa en la propia organización del rito, teniendo por tanto como consecuencia la caracterización espacial del lugar en que este se producía. El espacio, donde el vacío inicialmente era el protagonista, fue progresivamente adoptando una morfología concreta e incorporando significados que, hibridados al lugar, permanecen ligados al mismo como herencia inmaterial.

En las sociedades premodernas, cuando el concepto de individuo aún no existía, la persona se definía a través de sus relaciones con el resto del grupo (Vigliani 2016). Estas se generaban en un determinado lugar cuya configuración marcaba el modo en que estas relaciones se producían, generando unas cualidades intangibles que quedaban enlazadas a este. Así, los primeros espacios diseñados como enterramientos adoptaron formas circulares, como los "tholos", para mutar progresivamente a formas rectangulares, en un claro ejercicio de organización y, posteriormente, de jerarquización. De este modo, la geometría y el orden comenzaron a contemplarse como símbolos que debían regir la configuración espacial frente al desorden insondable de la naturaleza, como forma simbólica de conexión entre hombre y divinidad, un proceso cuya significación se acrecienta con el nacimiento y desarrollo de la metafísica y la progresiva toma de conciencia del individuo frente al grupo.

La forma en que las personas experimentan un espacio y las sensaciones que este genera están estrechamente relacionadas con la experiencia temporal y los vínculos sociales establecidos a través del hecho cultural (Grantham and Tuan 1978). Muy pronto, lo ritual y sagrado, como acto alejado de la vida ordinaria, comenzó a asociarse a espacios concretos separados de los hábitos y ámbitos cotidianos. Su clara escisión del espacio común quedaba representada mediante umbrales o puertas que simbolizaban 
esa transición. De igual modo, una determinada orientación, o la proximidad a elementos geográficos relevantes como ocurre con el dolmen de Menga en Antequera, eran cualidades que, asociadas a una determinada localización, tenían la propiedad de conceder un valor trascendental a lugares que, por ello, eran elegidos para el desarrollo de rituales. En estos, la conexión con lo trascendente era posible a través de la contemplación y la celebración de ceremonias tales como los ritos de paso. Algunos lugares adquirían tal relevancia que llegaban a convertirse en auténticos centros de peregrinaciones ya en época neolítica, como ocurre con Stonehenge (Balter 2008).

Paulatinamente, la incipiente arquitectura ligada a estos espacios comenzó a posicionarse como soporte simbólico para el rito, fundamentalmente por su capacidad para concentrar valores abstractos en un intento de dar respuesta a preguntas trascendentales, aquellas que sólo podían contestarse desde la inteligencia del espíritu (Barret 2010). Hasta el final de la Edad Media, espacio y tiempo mantenían una relación unívoca, de forma que el dónde estaba ineludiblemente unido al cuándo (Giddens 1999) y re-memorar significaba hacer referencia a un lugar, a un paisaje o a un espacio concreto. La posterior implantación de la visión cartesiana del mundo transformó la realidad en una dualidad constante, donde cada concepto se definía por oposición a otro, a la manera del tiempo y el espacio.

De la relevancia del espacio y la rotundidad de la materia ordenada conforme a cánones geométricos, en un tiempo cíclico donde no había aún conciencia de pasado o futuro, se pasó a la organización lineal del tiempo moderno, constriñendo el ámbito de lo sagrado a los espacios exclusivamente vinculados a la cuestión religiosa, apareciendo por oposición el espacio empírico alejado de toda divinidad (Berger 1971). Paralelamente, la concepción del hombre como ser individual supuso reafirmar la dualidad entre fe y razón, entre lo humano y lo divino. Se genera una nueva dimensión del ser humano a la que era posible acceder a través de la introspección. Este concepto del cuerpo como contenedor físico que atesora en su interior el alma iba a identificarse con los espacios considerados como sagrados, aquellos donde era posible la conexión con la divinidad a través del ritual, cuestión de especial relevancia en aquellos asociados al culto cristiano.

Por otra parte, si bien la vinculación con el envoltorio físico es un hecho, la relación establecida entre el ser humano y los lugares sagrados se ha producido de una multiplicidad de formas, pero siempre desde ese estado liminal y simbólico, donde es posible la conexión entre diferentes. Así ha ocurrido también con el paisaje, donde los elementos simbólicos de la geografía tienen un papel esencial en la configuración del espacio sagrado, como sucede habitualmente en los centros ceremoniales indígenas del continente americano, o bien el que se genera como vacío en núcleos y hechos urbanos complejos, donde cada elemento colabora en la composición de un espacio orgánico relevante, cuyo significado ha trascendido a lo largo del tiempo, como es el caso de Jerusalén, La Meca o Benarés (Albera and Couroucli 2012).

La concepción de un espacio como sagrado, no deja de ser una asignación de valores concretos que constituyen una apreciación subjetiva del hombre sobre la realidad transformada. Por ello, sin duda, es por lo que desde la arquitectura y su capacidad para generar significados encontramos la relación más estrecha con la dimensión espacial de lo sagrado.

\section{La fascinación por la arquitectura religiosa}

En su devenir histórico, como parte de su proceso de adaptación al medio, el hombre ha sentido la necesidad de fabricar objetos, siendo la arquitectura el más relevante y persistente de ellos. El espacio generado ha permitido el establecimiento de relaciones del hombre consigo mismo y con el propio medio, convirtiéndose en sí mismo en un valor a transmitir. De este dimanan los conocimientos acumulados, los discursos y pensamientos de cada periodo histórico, aprehensibles por aquellos que lo habitan y utilizan en épocas posteriores. Junto a la condición de inmanencia del espacio como derivada inherente a la composición material, resulta esencial su capacidad de impregnación de pensamientos y discursos, de relaciones e identidades, así como su trascedente capacidad narrativa, involucrando a la razón y a los sentidos. Así, con los primeros cultos en sociedades neolíticas, fue el espacio de la cueva el destinado a tales fines, trascendiendo del carácter de refugio a lugar en el que desarrollar las prácticas y ritos sagrados con el inicio de las primeras manifestaciones sociales del hombre, cuando geografía y religión formaban un binomio inseparable (Kong 1990).

La definición morfológica y tipológica del espacio se fue modificando en función de las necesidades simbólicas relacionadas con el culto, teniendo además las exigencias impuestas por las prácticas 
sociales durante la celebración del ritual un papel fundamental. Si en un principio, espacios y lugares se contemplaban como depositarios eternos de relatos sobre mitos y alegorías, fue la dualidad tiempo-espacio la que los transformaría en testigos indiscutibles de la historia y la memoria, prevaleciendo sobre lo material, convirtiendo los espacios en contenedores de identidades y valores. Como ejemplo de esto, el valor sagrado atribuido a la cúpula tiene su origen en el espacio de cobijo y protección que representaba la caverna primitiva (Moya Blanco 1978), la cual se revistió de mito como soporte de las primeras manifestaciones artísticas del hombre, evolucionando hasta simbolizar la bóveda celeste, pasando a considerarse como un espacio sagrado por su conexión con la divinidad (Koprinarov 2017). Este significado se ha transferido a las diferentes culturas y territorios a lo largo de la historia, perdurando de forma inconsciente hasta su vinculación moderna con la representación e imagen del poder del hombre en la tierra. Las formas circulares asociadas al espacio de cobijo y protección del grupo en los primeros abrigos fueron posteriormente adoptadas tanto para los primeros refugios artificiales como en las primeras tumbas. Estas, moradas para la eternidad en el seno de la madre tierra, eran construidas a partir de dólmenes sobre los que posteriormente se erigía un túmulo, formalizándose así como cuevas artificiales. Esta morfología asociada al círculo fue posteriormente sustituida por las formas geométricas ortogonales, como trasunto del orden y del control del hombre sobre el medio, cuestión de la que también se contagiarían el diseño y la propia concepción espacial.

El peso simbólico depositado sobre el espacio sagrado por las diferentes culturas, conforma una capa conceptual que pervive en lo heredado, transmitiéndose desde su creación a las generaciones venideras, reafirmando el valor del espacio como acumulador de significados invariantes a lo largo del tiempo.

Todo el sistema de pensamiento que da sentido al significado del espacio sustenta el tejido de identidades individuales y colectivas que refuerzan los vínculos entre los individuos y entre estos con el propio territorio y sus formas de apropiación. Distintas interpretaciones de la realidad elaboradas por diferentes culturas han materializado la envolvente arquitectónica de sus espacios sagrados en forma de templos, mezquitas, sinagogas y otros espacios destinados a acoger las necesidades espirituales y las creencias del grupo. Este, además de generar un lugar en el que desarrollar el rito, ha adquirido al mismo tiempo los valores intangibles que han posibilitado el vínculo y pertenencia a la comunidad y al lugar.

Ahora bien, la atribución de cualidades sagradas a un espacio a partir de su expresión material y constructiva es algo mucho más complejo; no supone únicamente un "imago mundi" del ritual vinculado al mismo, sino que también supone la reproducción terrenal de un modelo trascendente (Eliade 1959). El espacio adquiere así esa condición liminal, de umbral, que permite experimentar el tránsito de la dimensión real o exterior a la espiritual, a través de toda la carga emocional que las prácticas sociales y ritos han depositado a lo largo del tiempo. Espacialidad y materia, y con ellos los relatos y significados precipitados desde la elaboración de los primeros mitos y creencias, se interrelacionan para hacerse perceptibles, aún de una manera inconsciente en la experimentación y vivencia del espacio. Así, en religiones como el judaísmo, el cristianismo o el islam, el espacio sagrado adquiere un valor esencial como escenario donde las prácticas rituales generan espacios de reflexión y aprendizaje a través de la comunión entre los fieles y la divinidad, en un ejercicio que fortalece la identidad aportando seguridad al individuo en comunión con el grupo en medio de la incertidumbre y dispersión de su realidad cotidiana.

La individualidad como concepto humanista moderno, requiere del refuerzo de los valores del grupo como ejercicio para reforzar la separación del hombre frente a los demás y frente al mundo, haciendo patente su experiencia de socialización en el mundo físico (Proshansky 1978). Una parte importante y fundamental de esta identidad se define, en gran medida por el vínculo espiritual que, a través de las prácticas religiosas, se establece mediante su capacidad vivencial a través del espacio, donde se comparten sonidos, olores y representaciones estéticas, a través de las cuales es posible dilatar el "espacio de tiempo" para cruzar el umbral hacia lo espiritual. Esto además no está ligado exclusivamente a espacios interiores. Encontramos ejemplos paradigmáticos de lugares capaces de generar esta evocación tales como el Muro de las Lamentaciones de Jerusalén, los ya citados menhires de Stonehenge o las grandes pirámides de Giza, donde símbolo e imagen confluyen para actualizar nuestra memoria primitiva a través de la experiencia del espacio y su materialidad. De esta forma, al visitar lugares sagrados, muchas personas afirman que experimentan una sensación global que envuelve y alcanza a todos sus sentidos (Swan 1988). Atendiendo a este componente transpersonal 
y a las experiencias espirituales o emocionales capaces de ser generadas en la vivencia de espacios y lugares, nos hace comprender que, lejos de asociaciones con determinadas creencias, existe una componente adicional que dota y añade al espacio de cualidades representativas que completan su apariencia meramente física (Mazumdar and Mazumdar 1993).

La experiencia del espacio, que adquiere sentido con la arquitectura, nos conduce a reflexionar sobre la dimensión espiritual del ser humano y su capacidad para condensar en el instante presente toda la dimensión de la historia acumulada, más allá del continente material que lo conforma. La componente espiritual y emocional de un lugar destinado al culto, permanezca o no en uso, especialmente en aquellos templos que, además han conservado sus características y atractivos arquitectónicos, debe ser cognoscible y experimentada (Othman, Petrie, and Power 2013).

Con este marco de referencia, aproximarnos al concepto de Patrimonio desde un punto de vista genérico, supone tomar conciencia de que no sólo enfrentamos una realidad física, mueble o inmueble, sino que también condiciona nuestra mente, nuestra forma de ser y estar, a través de los recuerdos, vivencias y resultados de la vida en comunidad, que queda prendida en las construcciones y espacios públicos. Así, podremos referirnos al Patrimonio tangible e intangible que, en la manera en que está íntimamente ligado a la memoria colectiva, se expresa a través de sus edificaciones icónicas. Por ello, templos y edificios públicos o representativos se constituyen en símbolos de una identidad, elementos de cohesión cultural para la identificación de las comunidades que lo habitan.

La destrucción generada por el paso del tiempo o bien aquella devenida de los desastres naturales supera el valor económico de las construcciones, dañando irrecuperablemente la memoria social y cultural que representan, quedando también afectado su valor trascendente como herencia a transferir. Así, su mantenimiento y conservación preventiva supone la preservación del más importante legado cultural. Una muestra del desarraigo producido por la pérdida de elementos patrimoniales lo tenemos en Iquique (Chile) donde, tras el terremoto de Tarapacá en 2005, que produjo cuantiosas pérdidas de vidas humanas y edificios paradigmáticos como la Iglesia de San Lorenzo (1723) declarada Patrimonio de la Humanidad, los afectados llegaron a comentar al presidente del país en su visita a la zona "no me preocupa mi casa, pero quiero que me recupere mi Iglesia" (AFP 2005). Asimismo, en el estado mexicano de Oaxaca, tras el sismo de 1999, la mayoría de las comunidades rurales prefería que primero se reparasen los templos afectados que las propias casas de sus moradores, en ocasiones en peor estado de conservación e incluso con riesgo para las vidas de sus ocupantes (Sigler-Islas 2001).

Esta aprehensión del concepto de Patrimonio arquitectónico nos lleva a inferir la trascendencia que su conservación supone para la sociedad de la que emana, siendo tal la relevancia de los centros con valor espiritual, que incluso en aquellos casos en que se han superpuesto diferentes culturas sobre un mismo territorio en el tiempo, los espacios han mantenido su vocación para el mismo uso. El procedimiento habitual consistía en mantener la estructura y composición material, transformando su significado con pequeñas intervenciones para la adaptación a las nuevas necesidades. En casos extremos, podía demolerse la estructura anterior como ejemplo de sometimiento, siendo el lugar y el paisaje depositarios del valor y cualidades de antaño, aquellas que enaltecían el espacio para su reutilización. En otras ocasiones, el edificio colapsaba por su deterioro y envejecimiento, por su abandono o por la implicación de desastres naturales, como ocurrió con la actual Dominus Flevit de Jerusalén que se erige sobre los cimientos de la primitiva iglesia bizantina del siglo VII, de la que se conservan algunos mosaicos sobre el pavimento actual (Figura 2: Iglesia del "Dominus Flevit" en Jerusalén); previamente a la iglesia actual en ese mismo emplazamiento estuvo ubicada la mezquita El Mansuryeh, poniendo en relieve la capacidad de pervivencia de la memoria de los espacios y los vínculos emocionales que establece con los individuos durante las prácticas sociales. No en vano, es una evidencia que, por encima de la arquitectura que lo conforma, estos espacios litúrgicos ostentan el mayor porcentaje de visitas desde el punto de vista turístico, siendo su rehabilitación motivo de proyección internacional, por encima de las barreras culturales impuestas. En algunas actuaciones, el reacondicionamiento de espacios para su visita, cuenta con un nivel de aceptación social y un número de visitantes que trasciende el propio ámbito de la localidad donde se ubican, generando desplazamientos similares a la peregrinación (Jaramillo-Morilla, Ruiz-Jaramillo, y Mascort-Albea 2010).

En algunas ocasiones, la arquitectura se transforma para adaptar el espacio a nuevos usos que permitan la supervivencia del inmueble, manteniendo su potencial y valor espiritual intacto, a pesar de estar destinado a usos diferentes, evidenciando el arraigo emocional que el espacio conserva, 
independientemente de la materialidad que lo compone y que, sin duda, se interpreta como un soporte espiritual sobre el que se establece la identidad de la comunidad.

\section{Figura 2: Iglesia del "Dominus Flevit" en Jerusalén}
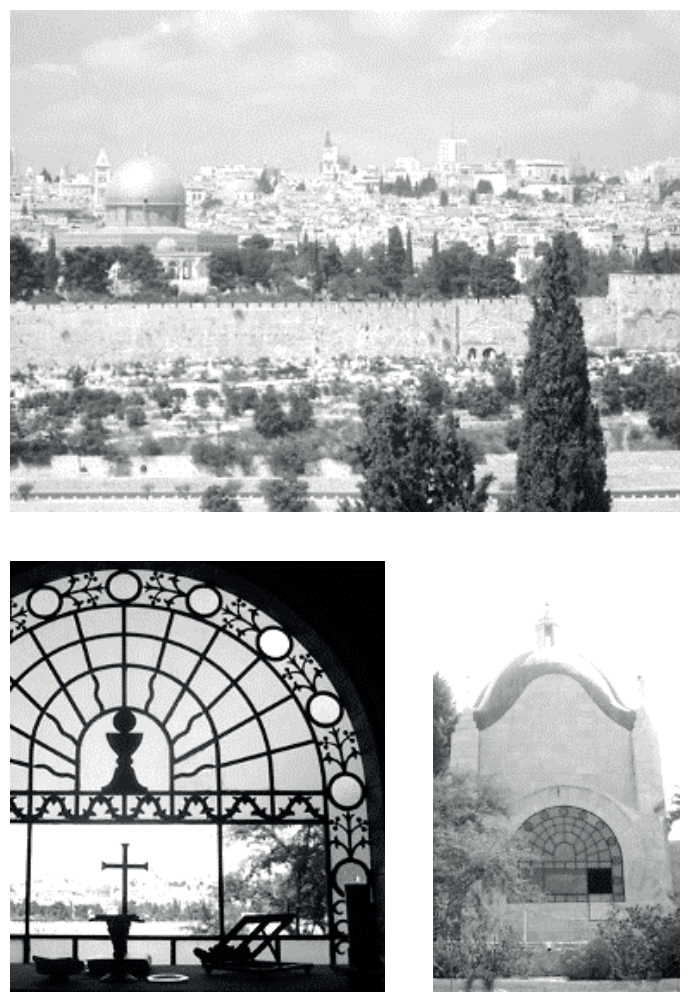

\section{La transformación de la ciudad contemporánea. El valor de lo común.}

Cualquier grupo social, independientemente de la complejidad de su organización, presenta unos valores concretos que lo mantiene unido y a través de los cuales se reconoce (Del Viso, Fernández Casadevante, and Morán 2017). Este tipo de nexos vinculados a lugares y espacios forman parte del campo de la etnología por su vinculación con hábitos y costumbres consolidados desde tiempos remotos, quedando hibridados a la tradición, y consecuentemente, mantenidos y preservados con mínimas transformaciones en el devenir del tiempo, fundamentando su trascendencia en su vinculación con materia y lugar. Por extensión, este valor asignado a lugares y espacios, que subyace en las prácticas sociales y discursivas, es común a cualquier grupo o comunidad que los comparte y cohesiona en la construcción de su identidad.

Con esta perspectiva sobre los lugares y paisajes y su valor patrimonial, podemos acometer la ciudad contemporánea y las diversas acciones de renovación implicadas en su mantenimiento, donde el patrimonio se posiciona como un valor esencial. Junto a la conservación de los conjuntos edificados en función de su estado, resulta determinante la implementación de medidas dirigidas a promover el mantenimiento preventivo y la recuperación de aquellos edificios con mayor interés, especialmente si se encuentran vinculados a un uso que permita garantizar su pervivencia en el tiempo.

Es evidente que la reconversión de los espacios para su acondicionamiento a otros usos de forma eficiente es una garantía para su puesta en valor y recuperación, conforme a las nuevas necesidades que demanda la sociedad en cada momento. Un claro ejemplo sobre esta disposición, lo constituye 
la intervención realizada en los Silos de Dorrego, en Buenos Aires (Figura 3: Recuperación para uso residencial de un antiguo silo en Buenos Aires, Argentina), cuyo su uso primigenio para el almacenaje de grano en la producción de harinas fue reconvertido en el año 1994 en un espacio para viviendas de la mano de MSGSS Arquitectos (Morales Méndez and Ruiz-Jaramillo 2008). Estos ejemplos de intervenciones, donde es posible la remodelación del espacio para adecuarlo a usos muy diferentes, muestran la imprescindible sensibilidad de los técnicos implicados en el proceso, capaces de mantener sus valores intrínsecos a pesar de la transformación del contenedor material.

\section{Figura 3: Recuperación para uso residencial de un antiguo silo en Buenos Aires, Argentina}
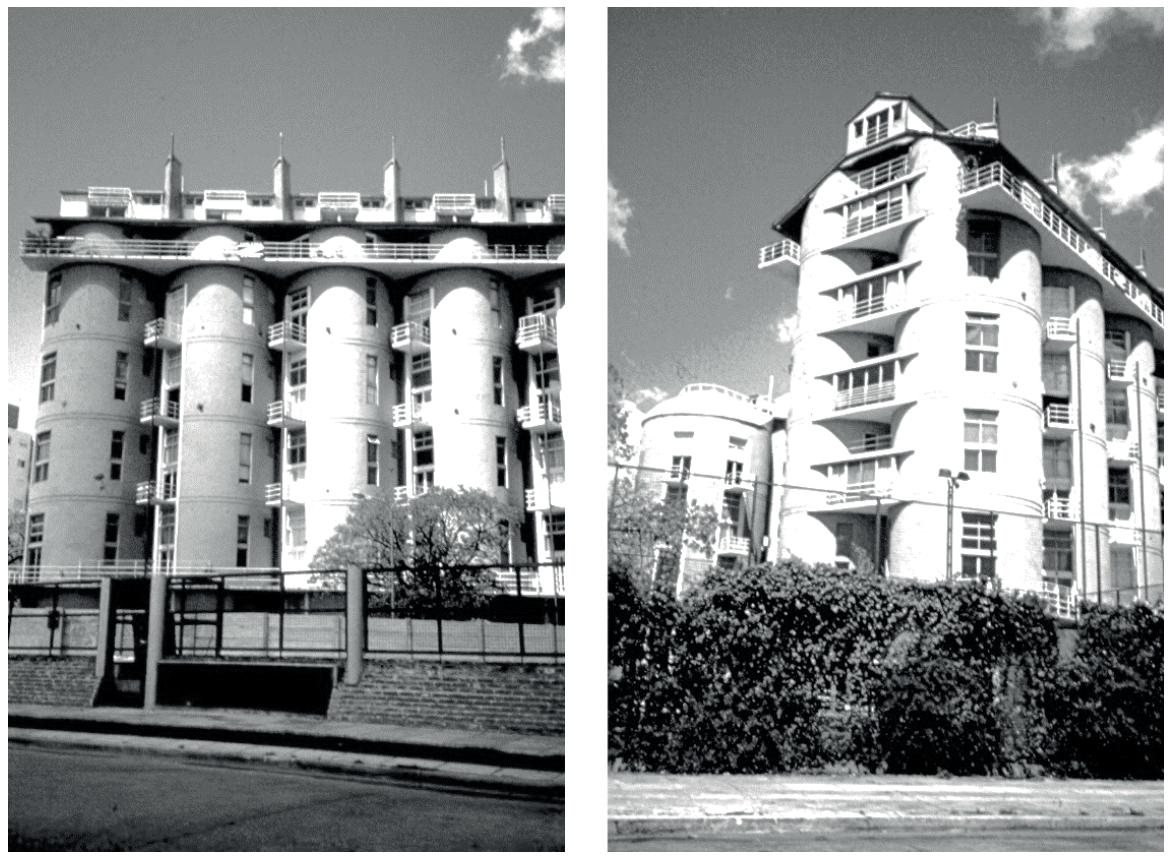

El interés por estas operaciones de remodelación y transformación se multiplican cuando trabajamos sobre edificios de carácter religioso, cuya permanencia en una sociedad cada vez más desvinculada de los ritos litúrgicos genera multitud de espacios con carácter sagrado que no se utilizan para los ritos y celebraciones religiosas. Como ejemplo de ello, en España el número de personas que nunca asiste a un oficio religioso ha pasado en cuarenta años de un 13 a un 56\% (CIS 1973, 2005, 2010). Como derivada de esta situación, que se extiende de manera generalizada en los países occidentales, muchos de estos espacios, cuya función a lo largo de la historia ha permanecido ligada a la celebración litúrgica, permanecen sin uso, en letargo, con falta evidente de las labores mínimas de mantenimiento, cuestión que tiene como consecuencia el abandono y la progresiva degradación de sus componentes y materiales.

La alternativa de continuidad que se plantea, como herramienta para la preservación del patrimonio, pasa necesariamente por revertir este proceso asignándoles un nuevo uso por parte de la comunidad como espacio vivencial, proponiendo un nuevo horizonte. Existen múltiples ejemplos de esta reconversión funcional en edificios como el Club Paradiso en Ámsterdam o el Gattopardo Caffé en la ciudad de Milán. En el primero de ellos, se remodeló un edificio del siglo XIX para albergar en su interior un club de baile, lugar en el que hasta 1965 se congregaba el grupo religioso liberal holandés Free Congregation. Por su parte, el Gattopardo Caffé se ubica desde el año 2001 en el interior de una antigua iglesia cristiana construida a inicios del siglo XX (Peric and Ravnic 2012).

Frente a esta transformación radical, sin embargo, es posible encontrar otros ejemplos de templos cuya reconversión ha resultado más amable en relación al uso trascendental para el que fueron 
concebidos. En estas actuaciones, los espacios de carácter sagrado se moldean y adaptan para acoger actividades sociales que mantienen en cierta forma sus cualidades espirituales, albergando museos, galerías de arte o bibliotecas, actividades donde la razón y la emoción permanecen presentes a lo largo de tiempos y espacios. Estas soluciones pasan fundamentalmente por asociar un destino cultural al lugar, entendiendo como cultura una forma de entender el mundo y adaptarse que singulariza a cada pueblo, permitiendo así dar continuidad a la vocación originaria que engendró ese lugar. La iglesia de Santa Lucía en Sevilla fue un templo cristiano hasta mediados de siglo XIX, que tras múltiples avatares y una rehabilitación realizada a comienzos del siglo XXI, ha sido abierta al público como centro cultural "IniciArte" (Figura 4a: Espacio "Iniciarte" en la iglesia de Santa Lucía en Sevilla; donde tienen lugar exposiciones de artistas emergentes andaluces, posibilitando la lectura del espacio sagrado como templo para el arte. De forma similar, se sitúa la intervención en la Selexyz Dominicaen de Maastrich (Figura 4b: Biblioteca en la Selexys Dominicaen de Maastrich.), utilizándose en este caso el espacio antes litúrgico como contenedor de libros desde el año 2005.

Figura 4: Izqda. Espacio "Iniciarte" en la iglesia de Santa Lucía en Sevilla; Dcha. Biblioteca en la Selexys Dominicaen de Maastrich.
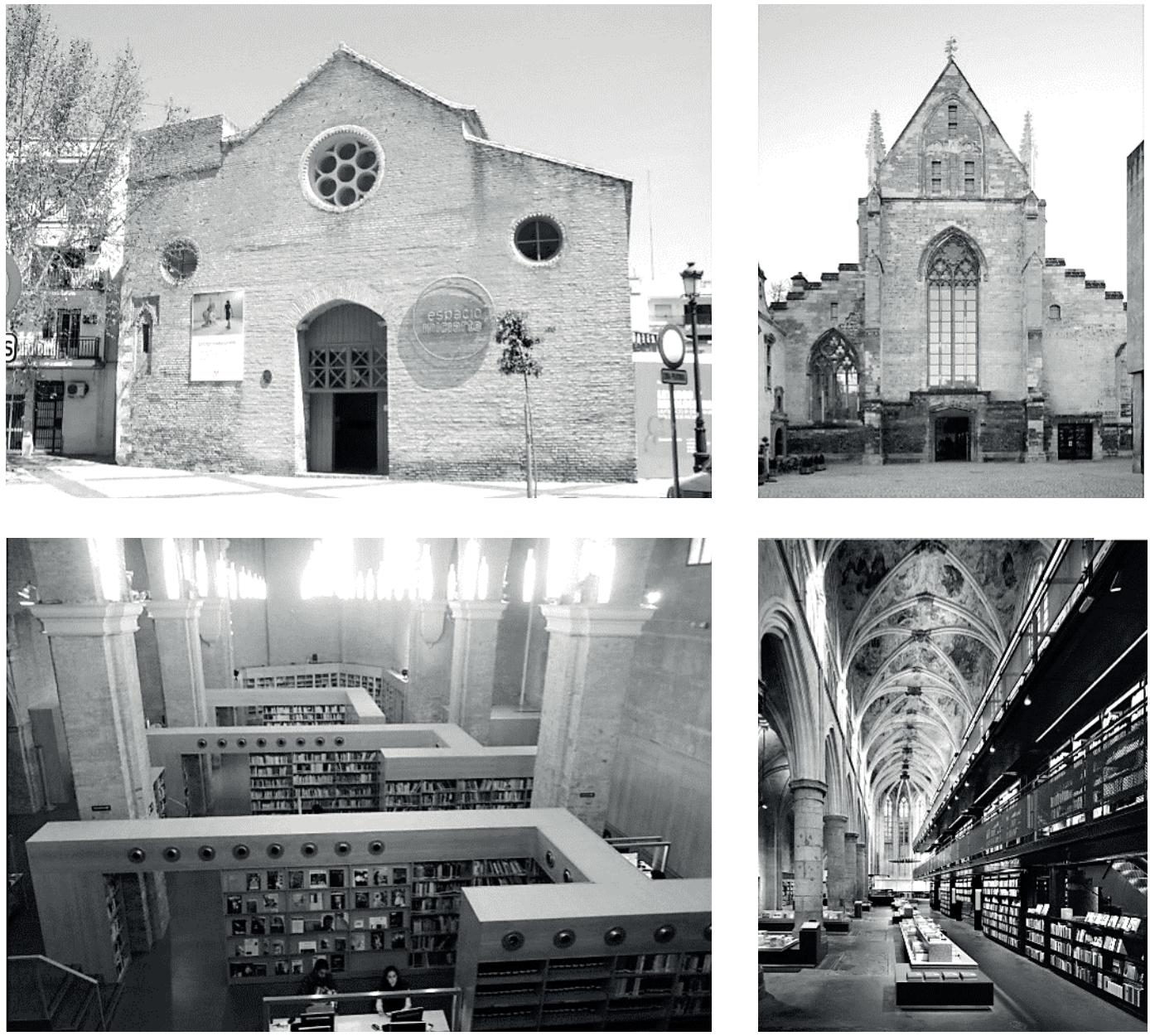

a 


\section{La transformación del espacio ritual a lo largo de la historia.}

Esta escueta visión que acaba de referirse sobre la reutilización de espacios de carácter religioso para usos contemporáneos se presenta como criterio a tener en cuenta al objeto de rehabilitar espacios de culto en una sociedad que cada vez se aleja más de las creencias trascendentales. Ahora bien, este fenómeno aparentemente ligado al desarrollo de la sociedad contemporánea viene produciéndose reiterativamente a lo largo de otras épocas históricas (Coomans 2012).

El hombre es un ser social que necesita de las relaciones "dividuales" (Vigliani 2016) para definirse a través de su capacidad para interactuar con los demás individuos y con el medio en que vive, donde resulta trascendental el modelo de sociedad y economía, así como su forma de concebir y pensar sobre la realidad. Del presente estricto de las antiguas sociedades se dio paso a una sociedad repleta de símbolos abstractos para imaginar lo desconocido (Márquez Muñoz 2017), con una nueva concepción del tiempo con pasado y futuro. Sin duda, una de las características más singulares es la necesidad y capacidad de relacionarse con otros individuos para colaborar, innovar y adelantarse a los acontecimientos, lo cual siempre implica de manera específica y concreta al espacio y a su envolvente arquitectónica. Sobre estas trazas se fundamenta la evolución del hombre y el desarrollo cultural en los diferentes espacios geográficos, incidiendo en la memoria del lugar y sobre la cronología de hechos, en la interrelación entre individuos para la construcción de una identidad.

La reutilización de espacios ancestrales para acoger otras prácticas sociales ha sido una forma constante de actuar, ponderando la presencia y protagonismo de espacios y lugares por su relevancia y permanencia. Así, desde la dominación romana y la generalización del cristianismo como religión de culto, la liturgia se encontraba hibridada a la adoración de divinidades paganas, como abstracciones de fenómenos inexplicables que se concretaban en lugares significados por la presencia de elementos relevantes de la naturaleza y por materialidades construidas por el hombre. A nivel global es posible hacer referencia a numerosos ejemplos de lugares y paisajes, sucesivamente incorporados a culturas donde la arquitectura ha compilado los significados, transmitiéndolos a lo largo del tiempo. Podemos destacar las actuaciones sobre el Partenón de Atenas (447-432 a.C.), el Panteón de Roma (118-125 d.C.), Templo romano de Antonino y Faustina (141 d.C.), Santa Sofía (360 d.C.), la mezquita de Córdoba (785 d.C.) o la sinagoga del Tránsito en Toledo (1360 d.C.). Este fenómeno se hace extensivo a los espacios litúrgicos de cualquier creencia religiosa.

Caso singular y paradigmático, en referencia a estas transformaciones de arquitecturas y lugares, resulta el sur peninsular español; sobre restos de antiguas edificaciones romanas, se construyeron los templos de la cultura visigoda destinada al culto cristiano. Posteriormente, con la entrada del islam, muchos de los espacios transferidos, fueron rehabilitados para el culto musulmán, reconvertidos nuevamente tras la conquista castellana y la expulsión de judíos y árabes.

\section{Un ejemplo de transformación y adaptación de espacios religiosos: el sur de la península ibérica}

Tras una larga etapa de dominación musulmana que se inició en el siglo VIII d.C., el siglo XIII trajo consigo un proceso de reconquista castellana en la zona del Bajo Guadalquivir, con la recuperación de importantes centros estratégicos como fueron las ciudades de Córdoba y Sevilla. Durante este proceso, el estilo gótico como culmen del cristianismo, se expandió por toda la península, encontrándose con un fuerte arraigo de la arquitectura islámica, que tuvo como consecuencia, un período de transición entre una y otra cultura; frente al predominio de la arquitectura musulmana, el gótico tuvo que hibridarse a la tradición imperante, generando un estilo particular de estos territorios denominado mudéjar (Amador de los Ríos 1872), para dar soporte a una población árabe que mantenía sus ritos y creencias en los territorios dominados por el acuciante cristianismo. Este estilo se convertirá en "el único tipo de construcción peculiarmente español de que podemos envanecernos" (Cómez Ramos 1974).

Dentro del territorio andaluz, este nuevo estilo tuvo una especial relevancia en las provincias de Córdoba y Sevilla, donde se utilizó para dar soporte y espacio a los nuevos usos representativos de las dos capitales destinados a un uso civil, como el Palacio de Pedro I en el Alcázar de Sevilla, siendo los templos destinados al culto los considerados como modelos del nuevo estilo. El patrón espacial cultural del cristianismo se transformó en base a una nueva configuración, donde simultáneamente convivían las cabeceras al estilo gótico con el cerramiento mural y la cubierta de madera de la tradición islámica. Así, la conversión de la mezquita a templo cristiano se produjo de una forma generalizada en todo 
el territorio reconquistado a los musulmanes, siendo en las ciudades de Sevilla, Córdoba y Granada donde podemos encontrar los ejemplos más característicos. Para llevar a cabo estas transformaciones se produjo la reconversión del tradicional espacio de la tradición musulmana mediante un sencillo proceso, que consistió en reorientar el eje configurador de su posición norte-sur a la este-oeste, consagrando el mihrab o altar sagrado islámico a una capilla para el culto de una advocación mariana. Este mismo procedimiento de modificación de la estructura y morfología del espacio de una a otra cultura fue empleado tanto en grandes espacios de oración como en templos de menor entidad dentro del núcleo urbano, trasladando asimismo su simbología a los ámbitos políticos y organizativos de la ciudad, como manifiesto de la dominación de una cultura sobre otra.

Ejemplos paradigmáticos de esto son la transformación de la mezquita de Ibn Addabas y la Mezquita Mayor en la ciudad de Sevilla, consideradas por sus dimensiones y capacidad de acogida como "mezquitas de los viernes" durante la época andalusí, que se transforman en la iglesia de El Salvador y Catedral, respectivamente tras la toma de la ciudad por las tropas castellanas en 1248. En el caso específico de la iglesia de El Salvador, el lugar de oración de la mezquita dio paso a la iglesia colegial barroca que hoy conocemos a través de un complejo proceso constructivo, durante el cual, el muro perimetral exterior que contenía el patio de abluciones y el alminar, fue transformado por el nuevo culto cristiano imperante, siendo además incorporado por el tejido urbano circundante aunque manteniendo su estructura básica original.

Figura 5: Planta de la mezquita de Ibn Addabás y superposición entre la planta de la mezquita y la del templo actual en la iglesia del Salvador de Sevilla.
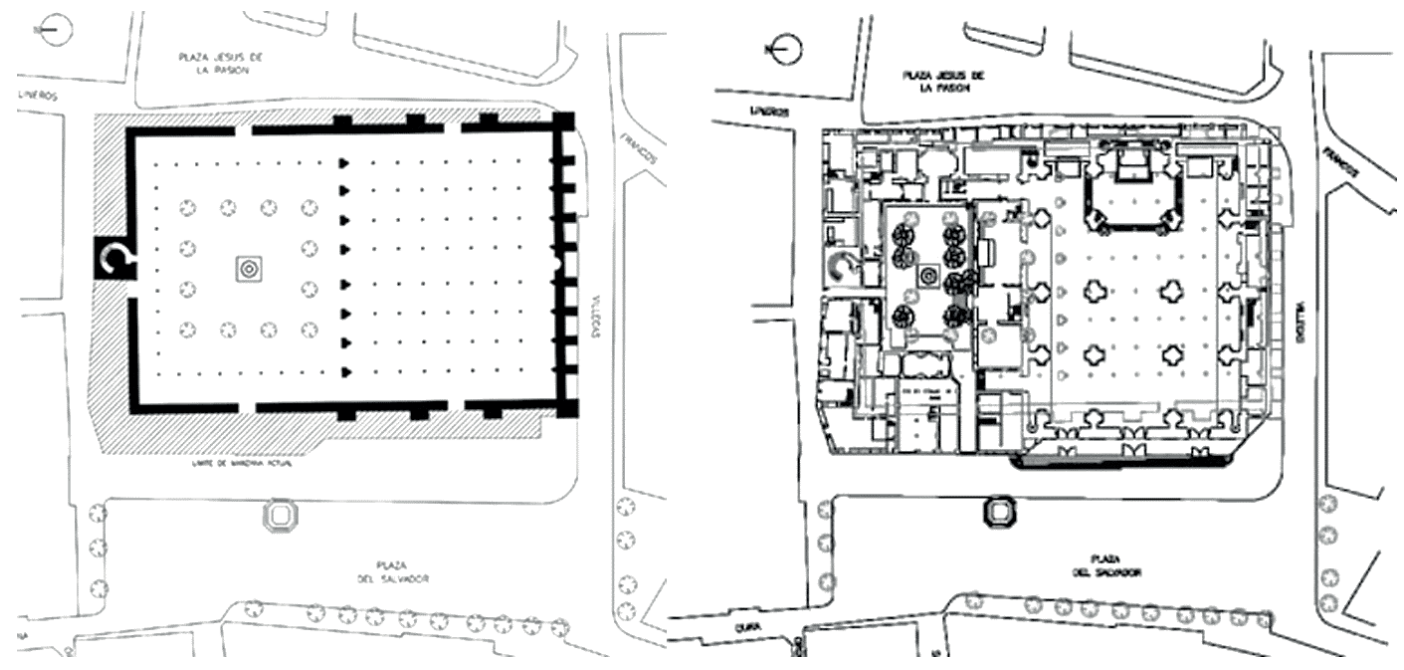

Este tipo de readaptaciones del espacio de las mezquitas en iglesias cristianas, manteniendo su funcionalidad (ambos continuaban siendo espacios litúrgicos) no fue algo exclusivo del cristianismo. En la mayoría de las ocupaciones militares se seguía un proceso similar ante la dificultad de repoblación integral de los nuevos dominios. Con el objeto de evitar que las ciudades ocupadas quedasen despobladas, era habitual mantener a sus habitantes, conservando costumbres e incluso sus prácticas religiosas, con la única salvedad de su sometimiento a impuestos y gravámenes especiales, a fin de lograr una progresiva y paulatina adaptación al nuevo orden social (Gómez de Terreros 2001).

En efecto, tomando como caso de estudio la propia ciudad de Sevilla, encontramos que a lo largo de la historia se ha venido produciendo de manera cíclica este proceso. Así, tras la ocupación árabe de la antigua ciudad visigoda (712-713 d.C.), se permitió la permanencia de la población cristiana y judía asentada previamente en la ciudad. Al igual que ocurriría con posterioridad con la reconquista castellana, la población fue asumiendo el islamismo al tiempo que muchos espacios religiosos se transformaron en mezquitas, ante la propia necesidad creciente de la población (Bosch Vilá 1988). Así, en el devenir de las diferentes culturas sobre un mismo territorio, espacio y materialidad se han mantenido por encima del devenir del tiempo, 
transformados y adaptados en función de los nuevos requerimientos de sus gentes. Tras esta primera conversión progresiva de espacios destinados al culto, se produjo su remodelación arquitectónica. Tomando de nuevo como objeto de análisis la iglesia de El Salvador de Sevilla en la que, como se ha comentado anteriormente, la antigua mezquita se transformó en colegial cristiana, el edificio readaptado sobrevivió durante más de cuatro siglos, hasta aproximadamente 1670, cuando hubo de ser derribada ante su avanzado estado de ruina. Este hecho supuso la construcción de un nuevo edificio sobre los restos materiales del anterior, empleándose para rellenar el hueco resultante del derribo de la mezquita por los propios escombros procedentes de su demolición sobre los que se inició la construcción de la nueva iglesia barroca.

Este proceso no es único de grandes edificios representativos. Hasta bien entrado el siglo XVI, los edificios en los que se encontraban mezquitas menores continuaron prácticamente inalterados, aunque transformado su uso en iglesia cristiana (Espinar Moreno and Quesada Gómez 1995) hasta que comenzaron a construirse los nuevos templos de forma paulatina. Este proceso, dependía de las posibilidades económicas del momento, por lo que en una primera fase parecía lógico el mantenimiento del primitivo edificio como medida de ahorro durante el período de tiempo necesario hasta contar con los recursos suficientes para abordar una nueva construcción. Otros parámetros debían tenerse en cuenta, más allá de la disponibilidad de recursos, como la capacidad de espacio que se requería para acoger a un incremento importante de población. Cuando se tomaba la decisión de demoler la mezquita existente, se acometía un trabajo gradual de marcado carácter simbólico; sobre los restos utilizados como firme, se trazaba el que sería nuevo ábside del templo para significar la consagración al nuevo culto. Posteriormente, y de nuevo, con una trascendental carga simbólica, se construía la portada de la nueva iglesia que daba acceso al espacio interior, a la dimensión espiritual, diseñada conforme al estilo gótico, aunque conservando los muros principales de la mezquita. Así, frente al adobe de los muros primitivos, se yuxtaponían dos elementos nuevos de cantería, manteniéndose el espacio como cuerpo central del templo y capilla mayor (Angulo Íñiguez 1983), teniendo especial cuidado en la trabazón entre los diferentes tipos constructivos. En la planta de la Figura 6 se han señalado los elementos constructivos adicionados a la mezquita preexistente (portada frontal y lateral, ábside, capilla lateral) o remodelados (torre) para acondicionar el espacio morfológica y funcionalmente al nuevo culto cristiano.

Queda así patente, el propósito de ir completando de forma paulatina el resto de la construcción, en el momento en que la disponibilidad de recursos lo permitiese, mediante una intervención estructurada en fases, que posibilitaba la continuación del uso del espacio, quedando en segundo plano, el continente material que lo conformaba. En numerosos casos, la ampliación o construcción del nuevo templo, no se produciría hasta que se encontrara en un estado de ruina, con la construcción apuntalada y presta a desplomarse (Angulo Íñiguez 1983), tal y como sucedía durante el reinado del monarca Pedro I, durante el que muchas de estas iglesias reconvertidas en mezquitas "permanecían en la humildad de sus principios" (Ortiz de Zuñiga 1677). Con el paso del tiempo, finalmente, el edificio primitivo, acabaría dando paso a su nueva imagen en la ciudad, conforme a los cánones establecidos por el nuevo gusto cultural, siendo el espacio y su función, los que permanecían.

\section{Figura 6: Alzado de la iglesia de Santa Marina (Sevilla); Planta con los principales elementos de la tipología arquitectónica de iglesia mudéjar sevillana; Ábside la iglesia de San Esteban (Sevilla)}
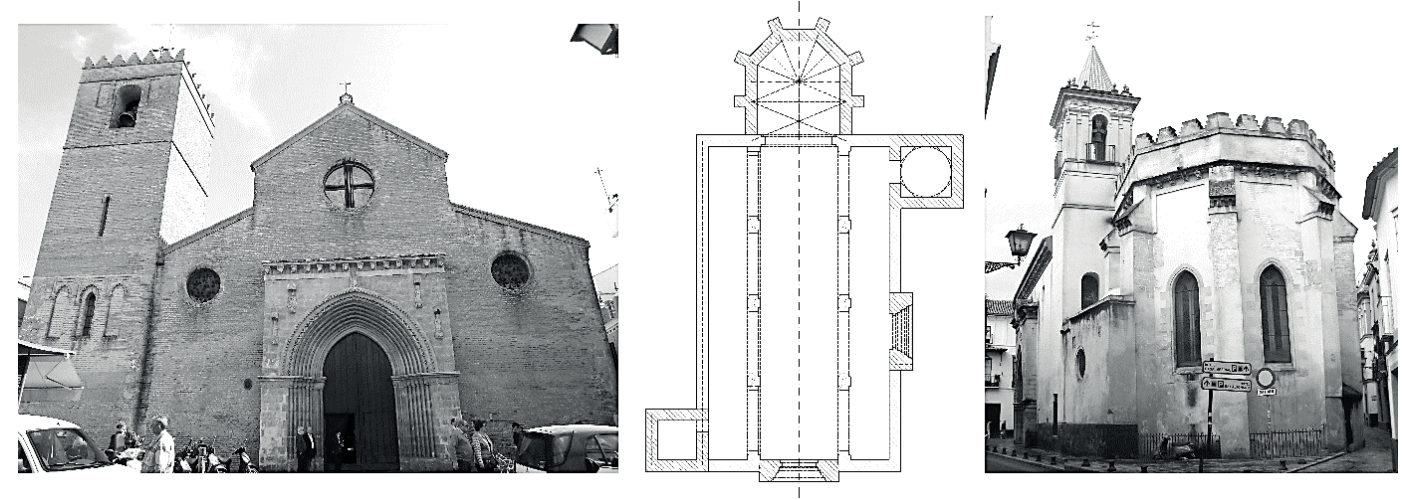


\section{Conclusiones}

Los espacios vinculados a valores inmanentes y trascendentes que suponen un referente para la identidad de la sociedad en que se insertan, más allá de su envolvente arquitectónica, presentan una serie de valores inmateriales que perviven a lo largo del tiempo, generando un valor añadido que resulta esencial respecto a la trascendencia de la arquitectura en la construcción del lugar y la identidad. Los lugares y espacios dotados con esas propiedades singulares, sagrados o vinculados a la espiritualidad, se han mantenido durante siglos en una continua evolución adaptativa a lo largo de la historia respecto a los requerimientos de culto o funcionales de diferentes liturgias y ritos, modificando su lenguaje arquitectónico, en mayor o menor medida. En esta continua evolución se han conservado estas características particulares que le atribuyen propiedades inmateriales por tanto asociadas al lugar, al espacio y no tanto al continente arquitectónico. En estas manifestaciones arquitectónicas generadas por el hombre, desde las primeras construcciones fusionadas con el paisaje hasta las elaboraciones contemporáneas más sofisticadas, se ha conservado el potente significado intrínseco en el espacio, entretejido con la propia identidad de la sociedad en que están inmersos.

Estas cualidades intangibles y abstractas, unidas al propio interés artístico y cultural vinculado directamente a la envolvente arquitectónica, presentan un enorme atractivo y fascinación para los visitantes desde épocas remotas. En efecto, desde las peregrinaciones a lugares sagrados de la antigüedad hasta el actual turismo de masas, estos valores intangibles que dimanan implícitamente de los espacios del patrimonio material que constituye la arquitectura, así como comprendidos en determinados paisajes, generan magnetismo y atracción, presentando la capacidad de conectar con ellos nuestra mente más primitiva, ofreciéndonos la posibilidad de aproximarnos a las claves de su origen, así como de su devenir a lo largo de diferentes épocas.

Por todo ello, el deterioro, destrucción y pérdida de este patrimonio no implica únicamente la pérdida de sus valores artísticos, culturales o paisajísticos. Su protección está conectada además a los valores trascendentes que identifican el lugar y la experiencia trascendentales de la comunidad inmanentes a estos espacios en los que la propia sociedad se identifica y acepta como suyos. La conservación de estos valores debe ser parte consustancial de cualquier proceso de renovación o rehabilitación que pueda producirse sobre el edificio, tanto si se trata de una actuación material o ligada a un cambio de uso, independientemente de que la nueva funcionalidad del edificio permanezca ligada a un uso litúrgico, unida por tanto al concepto de lo sagrado.

De esta forma, una concepción integral de los procesos de rehabilitación arquitectónica y urbana que integre y contemple el mantenimiento de estos valores, permitirá asegurar la conservación y transmisión, con todo su significado intrínseco, de los símbolos de identidad y elementos de cohesión cultural que identifican globalmente una sociedad.

\section{Bibliografía}

AFP. 2005. “Terremoto En Norte Chileno Causó Graves Daños Al Patrimonio Cultural.” Perú21. http:// peru21.pe/noticia/35760/terremoto-norte-chileno-causo-graves-danos-al-patrimonio-cultural (July 29, 2015).

Albera, Dionigi, and Maria Couroucli, eds. 2012. Sharing Sacred Spaces in the Mediterranean. Christians, Muslims, and Jews at Shrines and Sanctuaries. 1st ed. Cloth: Indiana University Press.

Amador de los Ríos, José. 1872. El Estilo Mudéjar En Arquitectura: Discurso. Facsimile. ed. Librerías París-Valencia. Valencia.

Angulo Íñiguez, Diego. 1983. Arquitectura Mudéjar Sevillana En Los Siglos XIII, XIV y XV. Ed. Facsím. Sevilla: Ayuntamiento de Sevilla.

Atakuman, Çiğdem. 2014. "Architectural Discourse and Social Transformation During the Early Neolithic of Southeast Anatolia." Journal of World Prehistory 27(1): 1-42.

Balter, Michael. 2008. "Early Stonehenge Pilgrims Came from Afar, with Cattle in Tow." Science 320(5884): 1704-5.

Barret, M. 2010. "The Monastery as Sacred Space." In Sacred Space: Interdisciplinary Perspectives within Contemporary Contexts, ed. 1st. Cambridge: Cambridge Scholars Publishing.

Berger, Peter L. 1971. El Dosel Sagrado : Elementos Para Una Sociologia de La Religión. 1st ed. Buenos Aires: Amorrortu. 
Bosch Vilá, J. 1988. Historia de Sevilla. La Sevilla Islámica: 712-1248. 1st ed. Seville, Spain: Universidad de Sevilla.

CIS. 1973. CIS Estudios 2752: Religiosidad. Madrid, España..

CIS. 2005. CIS Estudios 2630: Barómetro. Madrid, España.

CIS. 2010. CIS Estudios 2853: Barómetro. Madrid, España.

Cómez Ramos, Rafael. 1974. Arquitectura Alfonsí. 1st ed. ed. Diputación Provincial de Sevilla. Seville, Spain: Diputación Provincial de Sevilla.

Coomans, Thomas. 2012. "Reuse of Sacred Places: Perspectives for a Long Tradition." In Loci Sacri. Understanding Sacred Places, , 221-42..

Eliade, Mircea. 1959. The Sacred and the Profane the Nature of Religion. New York, USA: Harcourt Brace.

Espinar Moreno, Manuel, and Juan José Quesada Gómez. 1995. "Mezquitas convertidas en iglesias en las comarcas de Guadix y Baza (1490-1501): Datos sobre el urbanismo mudéjar." VI Simposio internacional de Mudejarismo, Teruel, 16-18 de septiembre de 1993 . Actas: 767-88.

Falk, John H., and Katie L. Gillespie. 2009. "Investigating the Role of Emotion in Science Center Visitor Learning." Visitor Studies 12(2): 112-32.

Giddens, Anthony. 1999. Consecuencias de La Modernidad. 1st ed. Madrid: Alianza Editorial.

Gómez de Terreros, Pedro. 2001. "Análisis Arquitectónico de Los Templos Parroquiales En La Ciudad de Sevilla: Santa Marina.” University of Seville.

Grantham, Charles E., and Yi-Fu Tuan. 1978. "Space and Place: The Perspective of Experience." Contemporary Sociology 7(4): 513.

Jaramillo-Morilla, Antonio, Jonathan Ruiz-Jaramillo, and Emilio Mascort-Albea. 2010. "Public Visits to Works in Built Heritage: A Necessity." In 2nd International Conference on Heritage and Sustainable Development, eds. Rogério Amoêda, Sérgio Lira, and Cristina Pinheiro. Évora, Portugal: Green Lines Institute for Sustainable Development, 1353-62.

Kong, Lily. 1990. "Geography and Religion: Trends and Prospects." Progress in Human Geography 14(3): 355-71.

Koprinarov, Lazar. 2017. "En Torno a La Espacialidad En Las Iglesias Cristianas y Mezquitas Musulmanas." Raphisa 1(2): 35-44.

Márquez Muñoz, Jorge Federico. 2017. "Génesis y Poder de Las Sociedades Primitivas. Berman y Girard.” Estudios Políticos 42: 37-52.

Mazumdar, Shampa, and Sanjoy Mazumdar. 1993. "Sacred Space and Place Attachment." Journal of Environmental Psychology 13(3): 231-42.

Mendoza-Castell, Fernando. 2008. La Iglesia Del Salvador de Sevilla: Biografía de Una Colegiata. 1st ed. Seville, Spain: Tebar.

Morales Méndez, Enrique, and Jonathan Ruiz-Jaramillo. 2008. "Centros Históricos y Edificación En El Patrimonio." In Arte y Tecnología. Lo Local En Lo Global, Buenos Aires: Academia Nacional de Bellas Artes, 47-66.

Moya Blanco, Luis. 1978. "Sobre El Sentido de La Arquitectura Clásica." In Tres Conferencias de Arquitectura, Madrid: Colegio Oficial de Arquitectos de Madrid, 8-29.

Ortiz de Zuñiga, Diego. 1677. Anales Eclesiásticos y Seculares de La Muy Noble y Muy Leal Ciudad de Sevilla, Metrópoli de Andalucía. Facsimile. Seville: Guadalquivir.

Othman, Mohd Kamal, Helen Petrie, and Christopher Power. 2013. "Visitors' Emotions, Touristic or Spiritual Experiences in Historic Churches: The Development of Church Experience Scale (CES)." Procedia - Social and Behavioral Sciences 97: 675-83.

Peric, Marko, and Mirna Ravnic. 2012. "Project Management Solution: Remodelling of Sacral Object for Tourism Purposes." In 21th Biennial International Congress Tourism \& Hospitality Industry 2012 - New Trends in Tourism and Hospitality Management, Opatija, Croatia: Faculty of Tourism and Hospitality Management, 21-29.

Proshansky, H. M. 1978. "The City and Self-Identity." Environment and Behavior 10(2): 147-69.

Real Academia Española. 2017. Diccionario de La Lengua Española. Edición de. Madrid: Real Academia Española.

Sigler-Islas, Eduardo, ed. 2001. Patrimonio y Sismos: Memoria Fotográfica de Los Sismos de 1999 En El Estado de Oaxaca. 1st ed. Mexico City, Mexico: Instituto Nacional de Antropología e Historia (INAH). Swan, J. 1988. "Sacred Places in Nature and Transpersonal Experiences." ReVision (10): 21-26. 
Vigliani, Silvina. 2016. "La Noción de Persona y La Agencia de Las Cosas. Una Mirada Desde El Arte Rupestre." Anales de Antropología 50(1): 24-48.

Del Viso, Nuria, José Luis Fernández Casadevante, and Nerea Morán. 2017. "Cultivando Relaciones Sociales. Lo Común y Lo 'Comunitario' a Través de La Experiencia de Dos Huertos Urbanos de Madrid.” Revista de Antropología Social 26(2): 473-81. 